\title{
一种基于二喹啉甲酸- $\mathrm{Cu}^{+}$显色反应的毛细管电泳 检测蛋白质的新方法
}

孟庆威, 郭否, 申 睿, 谢剑炜*

(军事医学科学院毒物药物研究所, 北京 100850 )

摘要 采用毛细管电泳法和蛋白质显色反应-二喹啉甲酸 $(\mathrm{BCA})$ 法, 结合微波辅助反应, 在 $60 \mathrm{mmol} / \mathrm{L}$ 嗍酸盐缓冲 液 ( $\mathrm{pH}$ 9.5) 中, 实现了对蛋白质的快速毛细管电泳分析检测。同时以 $\beta$-环糊精为包合添加剂, 实现了 $\mathrm{BCA}-\mathrm{Cu}^{+}$复 合物和游离 $\mathrm{BCA}$ 的分离, 从而在波长 $200 \mathrm{~nm}$ 处以测定特征生成的 $\mathrm{BCA}-\mathrm{Cu}^{+}$复合物来间接检测蛋白质, 其峰强度 比直接检测蛋白质自身吸收的峰强度提高了 2 个数量级。对于转铁蛋白、蓄麻毒素, 其线性范围分别为 $2 \sim 200$ $\mathrm{mg} / \mathrm{L}$ 和 $2 \sim 100 \mathrm{mg} / \mathrm{L}$, 检出限分别为 0.33 和 $0.37 \mathrm{mg} / \mathrm{L}$ 。将该方法成功地应用于第一届蓄麻毒素国际实验室间 比对测试的部分样品, 含量测定结果满意。

关键词: 毛细管电泳; 二喹啉甲酸;亚铜离子; 显色反应; $\beta$-环糊精; 蛋白质

中图分类号:O658 文献标识码 :A 文章编号 :1000-8713(2010)07-0682-06

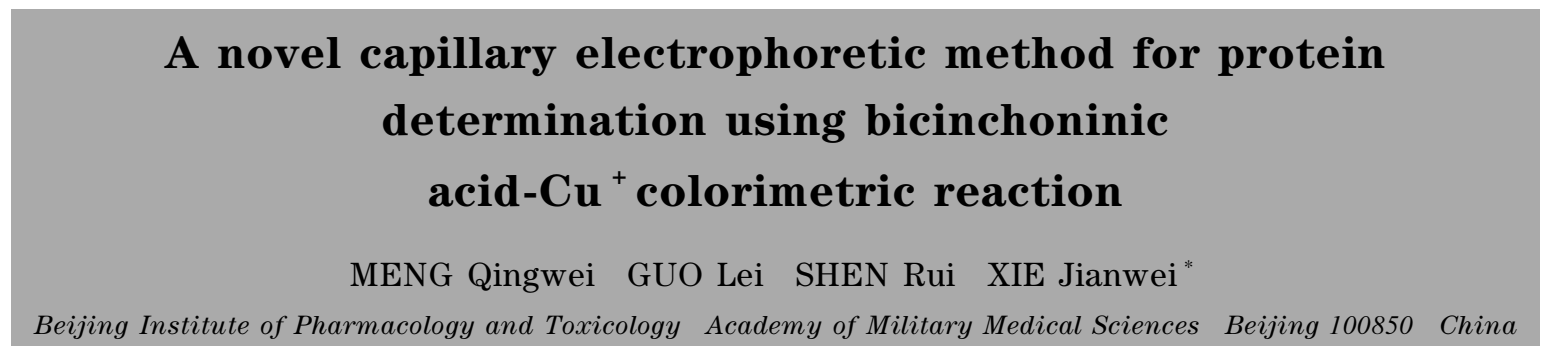

Abstract : A new rapid, sensitive method for protein determination using capillary electrophoresis and specific colorimetric reaction of bicinchoninic acid (BCA) was established, assisted by microwave incubation. With $60 \mathrm{mmol} / \mathrm{L}$ boric acid buffer $(\mathrm{pH} 9.5)$ and inclusion additive of $\beta$-cyclodextrin, the complex of $\mathrm{BCA}-\mathrm{Cu}^{+}$and free BCA molecules were efficiently separated. The peak intensity of $\mathrm{BCA}-\mathrm{Cu}^{+}$was higher than those of native proteins about two orders of magnitude at a low wavelength of $200 \mathrm{~nm}$. The linear ranges of this method were from 2 to 200 $\mathrm{mg} / \mathrm{L}$ for transferrin, and 2 to $100 \mathrm{mg} / \mathrm{L}$ for ricin. The limits of detection for transferrin and ricin were 0.33 and $0.37 \mathrm{mg} / \mathrm{L}$, respectively. This method was also successfully applied in the determination of some ricin samples in the First International Proficiency Test on the quantification of ricin.

Key words : capillary electrophoresis ( $\mathrm{CE}$ ) ; bicinchoninic acid ( BCA) ; $\mathrm{Cu}^{+}$; colorimetric reaction ; $\beta$-cyclodextrin ; protein

蛋白质是生命体行使生理功能的重要生物大分 子之一。针对功能蛋白质的高灵敏度检测新方法的 研究一直是生命科学领域的研究热点之一。针对蛋 白质的高灵敏度显色体系研究是生物传感,原位示 踪, 蛋白质/多肽药物的含量、纯度以及酶活力测定 等方面的基础 ${ }^{[1]}$,相关显色反应如 Bradford 法 (考

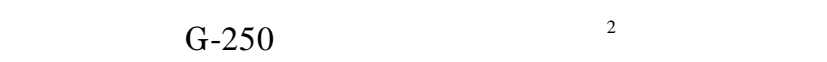
$\left(\mathrm{Cu}^{2+}\right.$ 与蛋白质的肽键在碱性条件下生成紫红色配 合物 ${ }^{[3]}$ 、Lowry 法( 蛋白质中的酪氨酸和色氨酸残 基与 Folin 试剂反应产生蓝色 $)^{[4]}$ 和二喹啉甲酸 (BCA) 法 ${ }^{[5]}$ 等已经在蛋白质分析中有大量的实际 应用。其中 BCA 方法的应用最为广泛 ${ }^{[5,6]}$ 。 
本文将毛细管电泳 (CE) 方法和 BCA 法结合起 来, 以 $\beta$-环糊精 $(\beta-\mathrm{CD})$ 为包合添加剂, 实现了 $\mathrm{BCA}$ $\mathrm{Cu}^{+}$复合物和过量游离 $\mathrm{BCA}$ 分子的分离。通过微 波辅助反应达到 $\mathrm{BCA}-\mathrm{Cu}^{+}$快速、完全显色的目的， 实现了在低波长处对蛋白质的高灵敏度检测。本方 法已成功地应用于第一届蓖麻毒素国际实验室间比 对测试蓄麻毒素蛋白样品的检测。

\section{1 实验部分}

\section{1 仪器和试剂}

$\mathrm{HP}^{3 \mathrm{D}} \mathrm{CE} 1100$ 型高效毛细管电泳仪 (美国惠普 公司) ,配备二极管阵列紫外检测器 (DAD)。内装 $50 \mu \mathrm{m}$ 内径的未涂层熔融石英毛细管 (金诺公司)。 有效分离长度和总长度分别为 $54 \mathrm{~cm}$ 和 $62.5 \mathrm{~cm}$ 。 系统控制和数据处理均由 HP Chemstation工作站 (版本号 B. 03.02) 完成。WP800SL23 型微波炉 (格 兰仕公司）,频率 $2450 \mathrm{MHz}$, 最大输出功率 1200 $\mathrm{W}$,使用额定输出功率 $420 \mathrm{~W}$; PHM-64 型 $\mathrm{pH}$ 计 (丹麦 Radiometer Copenhagen 公司) ; Cary 300 型紫外-可见分光光度计 (美国 Varian 公司)。实验 用超纯水 (电阻率 $18.2 \mathrm{M} \Omega \cdot \mathrm{cm}$ ) 均由 Mili- $\mathrm{Q}$ 型超 纯水仪制备 (美国 Millipore 公司)。

BCA、转铁蛋白 (transferrin)、L-丙氨酸 (L-Ala) 购自美国 Sigma-Aldrich 公司; 蓄麻毒素 (ricin) 由 本室提取纯化 ${ }^{[7]}$ 纯度 $>95 \% ; \beta$-环糊精 $(\beta$-CD) 、硼 酸、 $\mathrm{CuSO}_{4} 、 \mathrm{NaOH} 、 \mathrm{Na}_{2} \mathrm{CO}_{3} 、 \mathrm{NaHCO}_{3} 、 \mathrm{CuCl} 、$ 酒石酸 钾钠、二氧六环、 $\mathrm{POCl}_{3}$ 等均为国产分析纯试剂。

\section{2 溶液的配制}

取适量的转铁蛋白或蓄麻毒素溶于超纯水中 分别制得 $2 \mathrm{~g} / \mathrm{L}$ 的储备液。取适量的 BCA 及 $\mathrm{Cu}$ $\mathrm{SO}_{4}$ 溶于超纯水中, 配制浓度分别为 $200 \mathrm{mmol} / \mathrm{L}$ 和 $100 \mathrm{mmol} / \mathrm{L}$ 的储备液; 使用前, 取适量并以硼酸 盐缓冲液 ( $\mathrm{pH} 9.5$ ) 稀释至所需的浓度 (其中嗍酸的 最终浓度为 $10 \mathrm{mmol} / \mathrm{L}$ ), 并经 $0.45 \mu \mathrm{m}$ 水相微孔 滤膜 (美国 Millipore 公司) 过滤。硼酸盐缓冲液 $(20 \sim 300 \mathrm{mmol} / \mathrm{L})$ 用 $1 \mathrm{~mol} / \mathrm{L} \mathrm{NaOH}$ 溶液调至所 需的 $\mathrm{pH}$ 值。常规的 BCA 法使用的试剂包括碱性 储备液 ( $10 \mathrm{~g} \mathrm{Na}_{2} \mathrm{CO}_{3}, 2 \mathrm{~g} \mathrm{NaOH}, 4.75 \mathrm{~g} \mathrm{NaHCO}_{3}$, $0.8 \mathrm{~g}$ 酒石酸钾钠, 将其定容至 $100 \mathrm{~mL}$, 室温下保 存)、 $50 \mathrm{~g} / \mathrm{L} \mathrm{BCA}$ 溶液 ( $4{ }^{\circ} \mathrm{C}$ 下保存) 及 $40 \mathrm{~g} / \mathrm{L} \mathrm{Cu}$ $\mathrm{SO}_{4}$ 溶液。使用时将碱性储备液、 BCA 溶液( 50 $\mathrm{g} / \mathrm{L})$ 和水以体积比 $1: 1: 3$ 的比例混匀, 然后再与 40 $\mathrm{g} / \mathrm{L} \mathrm{CuSO}_{4}$ 溶液以体积比 50:1 混合, 即得到 $\mathrm{BCA}$ 法检测蛋白质所用的试液, 使用时将其 $\mathrm{pH}$ 调至 $\mathrm{pH}$ 9.5 。

\section{$1.3 \mathrm{CE}$ 条件}

所有 $\mathrm{CE}$ 实验中，毛细管首次使用前均依次用 1 $\mathrm{mol} / \mathrm{L} \mathrm{NaOH}$ 和水分别冲洗 $30 \mathrm{~min}$ 和 $5 \mathrm{~min}$ 以活 化毛细管壁。每次进样前，依次用 $0.1 \mathrm{~mol} / \mathrm{L}$ $\mathrm{NaOH}$ 、水及缓冲液分别冲洗毛细管 $3 \mathrm{~min}$ 。样品在 $5 \mathrm{kPa}(50 \mathrm{mbar})$ 压力下进样 $10 \mathrm{~s}$ 后, 于 $25 \mathrm{kV} 、 25$ ${ }^{\circ} \mathrm{C}$ (空气动力控温模式)下进行分离。DAD 检测波 长设为 $200,280,330$,400 及 $567 \mathrm{~nm}$ 。每天使用完 毕后，依次用 $0.1 \mathrm{~mol} / \mathrm{L} \mathrm{NaOH}$ 、水和空气分别冲洗 毛细管 $5 \mathrm{~min}$ 后关机。

\section{4 紫外-可见光谱 (UV-VIS) 测定}

在 $0.5 \mathrm{~mL}$ Eppendorf 管中, 按所需浓度移取一 定体积的 $\mathrm{BCA} 、 \mathrm{CuSO}_{4}$ 和蛋白质储备液, 用水补足 至 $100 \mu \mathrm{L}$,再准确移取 $100 \mu \mathrm{L}$ 浓度为 $20 \mathrm{mmol} / \mathrm{L}$ 硼酸盐溶液, 样品涡旋混匀后, 在 $37{ }^{\circ} \mathrm{C}$ 下孵育 30 $\min$ 后 转入 $1 \mathrm{~cm}$ 样品池中, 在 $200 \sim 700 \mathrm{~nm}$ 范围 扫描。为清楚起见，图 1 中仅给出 $415 \sim 670 \mathrm{~nm}$ 部 分的光谱图。

\section{5 丙均肽的合成}

丙均肽 $\left[(\mathrm{Ala})_{n}\right.$ ] 的合成参照文献 [ 8 ] 进行。取 含 $10 \mathrm{mmol} / \mathrm{L} \mathrm{L}-\mathrm{Ala}$ 与 $10 \mathrm{mmol} / \mathrm{L} \mathrm{POCl}_{3}$ 的二氧六 环溶液 $30 \mathrm{~mL}$, 在室温下磁力搅拌 $96 \mathrm{~h}$ 后, 用 $10 \mathrm{~mL}$ 水终止反应, 得到黄色油状液体, 即为丙均肽。经正 离子电喷雾质谱 (ESI-MS) 鉴定其为 $n<10$ 的丙均 肽混合物, 其 $m / z$ 为 $161.0933(n=2), 303.0612$ $(n=4)$, 374. $1455(n=5), 516.2473(n=7)$, $658.2573(n=9)$ 和 $729.3500(n=10)$ 。

\section{6 微波反应}

移取 $5 \mu \mathrm{L} \mathrm{BCA}$ 储备液、 $1 \mu \mathrm{L} \mathrm{CuSO}_{4}$ 储备液及 一定体积的蛋白质储备液置于密闭的 $1 \mathrm{~mL}$ 玻璃样 品瓶中，用水补足 $50 \mu \mathrm{L}$ 。再加入 $50 \mu \mathrm{L} 20 \mathrm{mmol} / \mathrm{L}$ 嗍酸盐溶液, 涡旋混匀后, 在额定输出功率 $420 \mathrm{~W}$ 下反应 $5 \mathrm{~min}$ 取出后立即置于 $4{ }^{\circ} \mathrm{C}$ 环境中放置 10 min 然后进行分析。

\section{2 结果与讨论}

\section{1 蛋白质显色反应的 UV-VIS 行为}

BCA 显色反应的原理 :在碱性条件下, $\mathrm{Cu}^{2+}$ 离 子与蛋白质中的还原性氨基酸残基 (如色氨酸、酪 氨酸、胱氨酸、半胱氨酸残基等) 或蛋白质中的肽 键 ${ }^{[9]}$ 发生氧化还原反应, $\mathrm{Cu}^{2+}$ 自身被还原成为 $\mathrm{Cu}^{+}$ 离子, 而 $\mathrm{Cu}^{+}$离子与 $\mathrm{BCA}$ 反应生成紫色产物, 最大 吸收波长为 $567 \mathrm{~nm}$ 。

BCA 法一般选择 $\mathrm{pH} 10 \sim 11$ 的碳酸盐缓冲液， 而采用 $\mathrm{CE}$ 分析蛋白质时，一般首选高压电场下稳 
定性和生物相容性好的硼酸/嗍砂体系作为其运行 缓冲液。因此, 本文首先比较了蛋白质 BCA 显色反 应在嗍酸盐缓冲体系和碳酸盐缓冲体系中的结果， 如图 1 所示。

在 $10 \mathrm{mmol} / \mathrm{L}$ 嗍酸盐缓冲液 $(\mathrm{pH} 9.5)$ 中, 当 $\mathrm{Cu}^{2+}$ 离子和 $\mathrm{BCA}$ 体系共存时,在 $500 \mathrm{~nm}$ 以上波长 下仅产生微弱的吸收（见图 1c) ;当加入蛋白质后， 由于形成复合物 $\mathrm{BCA}-\mathrm{Cu}^{+}$后于 $567 \mathrm{~nm}$ 处呈现出最 大的吸收 (见图 $1 \mathrm{~d}$ ) , 其摩尔吸光系数约为 $2.2 \times 10^{4}$ $\mathrm{L} /(\mathrm{mol} \cdot \mathrm{cm})$ 。对于碳酸盐缓冲盐体系, 其加入蛋 白质前后的显色行为 (见图 $1 \mathrm{e}$ 和 $\mathrm{f}$ ) 基本与嗍酸盐 缓冲液相似 (图 $1 \mathrm{c}$ 和 $\mathrm{d}$ )。在硼酸盐缓冲液中加入 固定浓度的蛋白质后, 随着 $\mathrm{BCA}$ 及 $\mathrm{Cu}^{2+}$ 浓度的增 大，在 $567 \mathrm{~nm}$ 处的吸光度值增加，当 $\mathrm{BCA}$ 浓度大于 $10 \mathrm{mmol} / \mathrm{L}$ 后吸光度增加不明显; $\mathrm{Cu}^{2+}$ 浓度大于 1 $\mathrm{mmol} / \mathrm{L}$ 时,体系产生沉淀。

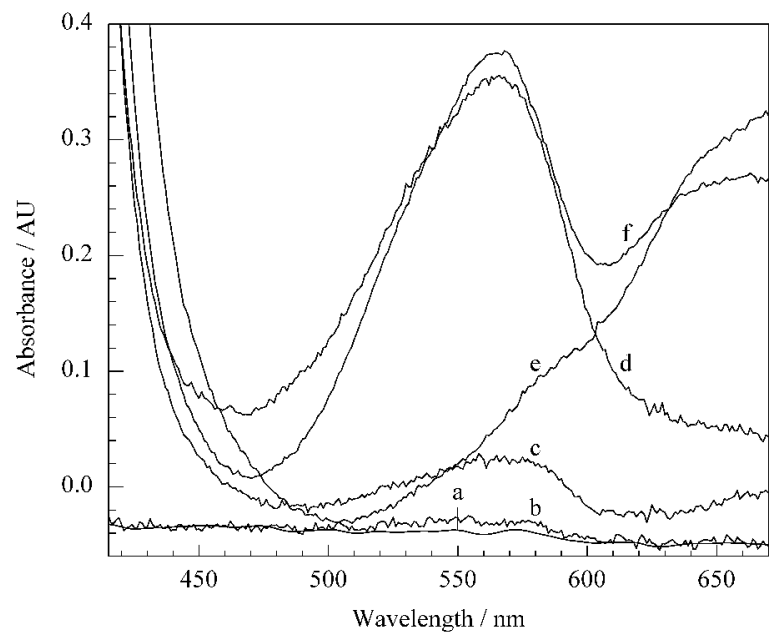

图 1 BCA 显色反应的紫外-可见光谱图

Fig. 1 UV-visible spectra of $\mathrm{BCA}-\mathrm{Cu}^{+}$complex in BCA colorimetric method

a. borate buffer as a blank ; b. BCA in borate buffer ; c. BCA and $\mathrm{CuSO}_{4}$ in borate buffer ; d. $\mathrm{BCA}, \mathrm{CuSO}_{4}$ and transferrin in borate buffer ; e. BCA and $\mathrm{CuSO}_{4}$ in carbonate buffer ; f. BCA , $\mathrm{CuSO}_{4}$ and transferrin in carbonate buffer. The above samples were measured at $\mathrm{pH} 9.5$ after incubation at $37{ }^{\circ} \mathrm{C}$ for $30 \mathrm{~min}$.

The final concentration of each component : borate buffer , 10 mmol/L ; carbonate buffer , $30 \mathrm{mmol} / \mathrm{L}$; $\mathrm{CuSO}_{4}, 1 \mathrm{mmol} / \mathrm{L}$; $\mathrm{BCA}, 10 \mathrm{mmol} / \mathrm{L}$; transferrin , $100 \mathrm{mg} / \mathrm{L}$;

\section{2 微波反应}

采用 $\mathrm{BCA}$ 法检测蛋白质时, 通常需要较高的温 度和较长的时间 ${ }^{[9]}$ 。由于其属于慢反应进程, 随着 放置时间的增加，吸光度值也不断地增大，从而导致 测定结果偏差较大。因此, 本文采用微波加速反应, 以达到快速、完全反应的目的 ${ }^{[10]}$ 。

采用 $\mathrm{BCA}$ 法测定蛋白质时，一般在 $37{ }^{\circ} \mathrm{C}$ 这样 较低的温度下,蛋白质中还原性氨基酸残基首先将
$\mathrm{Cu}^{2+}$ 还原为 $\mathrm{Cu}^{+}$, 随着反应温度提高至 $60{ }^{\circ} \mathrm{C}$,蛋白 质中的肽键对体系中的 $\mathrm{Cu}^{2+}$ 还原为 $\mathrm{Cu}^{+}$的贡献增 $大^{[6,9]}$ 。

在 $10 \mathrm{mmol} / \mathrm{L}$ 的硼酸盐缓冲体系 $(\mathrm{pH} 9.5)$ 中， 采用中等强度的微波输出功率 ( $420 \mathrm{~W}$ ) 对高 ( 100 $\mathrm{mg} / \mathrm{L}) 、$ 、 $(50 \mathrm{mg} / \mathrm{L})$ 和低 $(20 \mathrm{mg} / \mathrm{L}) 3$ 个质量浓度 的转铁蛋白进行微波反应, 结果如图 2 所示。其吸 光度随着反应时间的增加而稳步增加, 反应 $5 \mathrm{~min}$ 后, 其 $567 \mathrm{~nm}$ 处的吸光度值是相同条件下未经微 波处理在 $37{ }^{\circ} \mathrm{C}$ 孵育 $30 \mathrm{~min}$ 后的 7 倍。微波反应后 样品在室温下放置 $48 \mathrm{~h}$, 其吸光度值基本无变化, 表明采用微波加热可有效地加速反应, 并获得稳定 的测定结果。

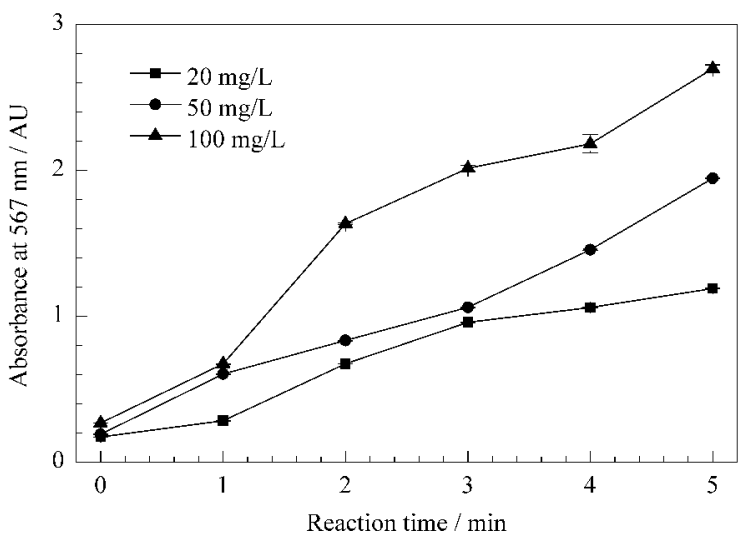

图 2 微波反应下不同浓度的铁蛋白的吸光度值变化

Fig. 2 Absorbances of transferrin with different concentrations after microwave-assisted incubation

为了比较微波反应 $(420 \mathrm{~W}, 5 \mathrm{~min})$ 和 $37{ }^{\circ} \mathrm{C}$ 孵 育 $30 \mathrm{~min}$ 两种条件对反应的影响, 分别以转铁蛋 白、色氨酸 (代表蛋白质的还原性氨基酸)、丙氨酸 (阴性控制) 及丙均肽 (代表蛋白质间的肽键) 进行 反应。结果表明, 在微波反应条件下, 由丙均肽还原 产生的 $\mathrm{BCA}-\mathrm{Cu}^{+}$峰高明显增高 (见图 3c-1 和 $\mathrm{c}-2$ ) , 说明在微波反应条件下, 肽键对 $\mathrm{BCA}-\mathrm{Cu}^{+}$反应的贡 献增加。

\section{3 基于 BCA 显色反应的 CE 快速检测蛋白质}

大多数蛋白质为碱性和中性蛋白质，例如转铁 蛋白 $(\mathrm{p} I=6.6)$ 和萫麻毒素 $(\mathrm{p} I=7.1)$, 虽然在本文 选择的高 $\mathrm{pH}$ 值 $\mathrm{CE}$ 体系下均带负电荷，但仍会通过 其疏水作用在毛细管壁上产生吸附 ${ }^{[11]}$ 。而采用 BCA 法处理蛋白质后，实际运行时仅对其特征生成 的 $\mathrm{BCA}-\mathrm{Cu}^{+}$进行检测，蛋白质在 $\mathrm{CE}$ 管壁中的吸附 因此可以忽略。

\subsubsection{CE 条件的优化}

本文选择样品缓冲液的浓度低于分离缓冲液的 


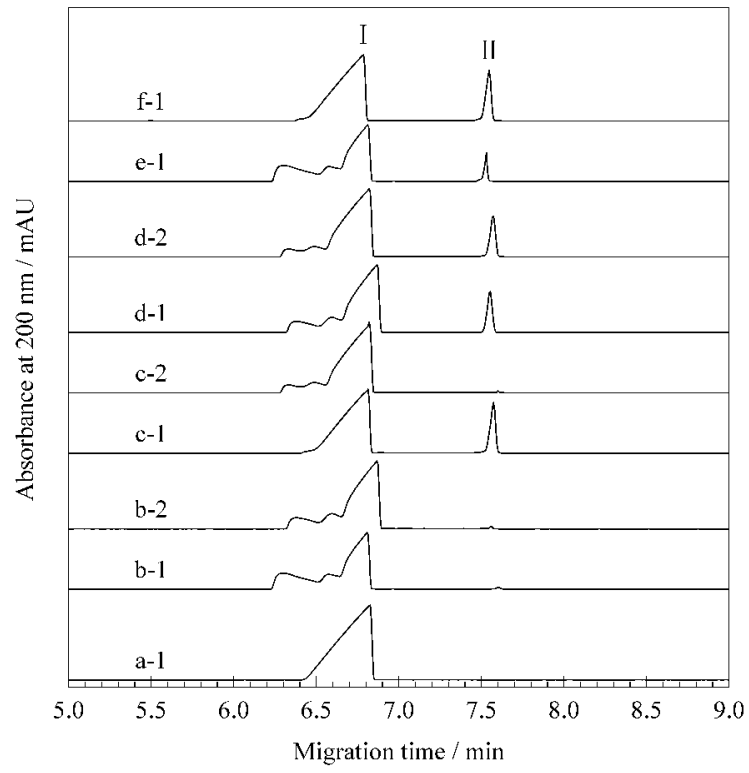

图 3 (1) 微波反应和 $(2) 37{ }^{\circ} \mathrm{C}$ 捊育 $30 \mathrm{~min}$ 两种条件下 BCA-Cu ${ }^{+}$复合物吸光度的变化

Fig. 3 Absobances at $200 \mathrm{~nm}$ for $\mathrm{BCA}-\mathrm{Cu}^{+}$complex under the conditions of (1) microwave operation and (2) incubation at $37{ }^{\circ} \mathrm{C}$ for $30 \mathrm{~min}$

CE injection buffer : $10 \mathrm{mmol} / \mathrm{L}$ borate buffer $(\mathrm{pH} \mathrm{9.5)} \mathrm{;} \mathrm{CE}$ separation buffer : $60 \mathrm{mmol} / \mathrm{L}$ borate buffer ( $\mathrm{pH} 9.5$ ) containing $10 \mathrm{mmol} / \mathrm{L} \beta$-CD.

a. $\mathrm{BCA}$; b. $\mathrm{BCA}+\mathrm{CuSO}_{4}+$ alanine ; c. $\mathrm{BCA}+\mathrm{CuSO}_{4}+$ $(\mathrm{Ala})_{n} ; \mathrm{d} . \mathrm{BCA}+\mathrm{CuSO}_{4}+$ tryptophan ; e. $\mathrm{BCA}+\mathrm{CuCl} ; \mathrm{f}$. $\mathrm{BCA}+\mathrm{CuSO}_{4}+$ transferrin. Concentration of each component : $\mathrm{BCA}, 10 \mathrm{mmol} / \mathrm{L}$; $\mathrm{CuSO}_{4}$ and $\mathrm{CuCl}, 1 \mathrm{mmol} / \mathrm{L}$; alanine, (Ala ${ }_{n}$, tryptophan and transferrin , $100 \mathrm{mg} / \mathrm{L}$.

Peak I : free BCA ; peak II : $\mathrm{BCA}-\mathrm{Cu}^{+}$.

浓度, 利用电场聚焦效应引起的样品堆积, 提高检测 灵敏度 ${ }^{[12]}$,也可有效避免样品缓冲液，即 BCA 法的 反应体系中硼酸盐浓度过高而引起的 $\mathrm{Cu}(\mathrm{OH})_{2}$ 沉 淀。当嗍酸盐浓度大于 $10 \mathrm{mmol} / \mathrm{L}$ 时,由于碱性条 件下大量 $\mathrm{B}(\mathrm{OH})_{4}^{-}$离子的存在 ${ }^{[13]}, \mathrm{Cu}(\mathrm{OH})_{2}$ 沉淀 形成的可能性增加, 不利于均相样品反应体系的形 成。另外, 正如前面提到的, 当 $\mathrm{Cu}^{2+}$ 离子浓度大于 $1 \mathrm{mmol} / \mathrm{L}, \mathrm{pH}$ 大于 9.5 时,也容易产生沉淀。样 品缓冲液中，随着 $\mathrm{BCA}$ 和 $\mathrm{Cu}^{2+}$ 的浓度分别增大至 $10 \mathrm{mmol} / \mathrm{L}$ 和 $1 \mathrm{mmol} / \mathrm{L}$ 时, 复合物信号值增加, 直 至保持最大值不变。因此我们选择的样品缓冲液为 $10 \mathrm{mmol} / \mathrm{L}$ 硼酸盐 $(\mathrm{pH} 9.5), 10 \mathrm{mmol} / \mathrm{L} \mathrm{BCA}$ 及 1 $\mathrm{mmol} / \mathrm{L} \mathrm{CuSO}_{4}$ 。

考察了不同分离缓冲盐浓度及 $\mathrm{pH}$ 值对复合物 峰高的影响。复合物峰随着硼酸盐缓冲液浓度的增 大而升高，但当嗍酸盐缓冲液浓度大于 $60 \mathrm{mmol} / \mathrm{L}$ 后, 复合物峰的峰高呈现降低趋势。复合物峰随着 $\mathrm{pH}$ 升高而升高, 当 $\mathrm{pH}>9.5$ 后, 复合物峰的峰高呈 现略微降低的趋势。因而最终选择 $60 \mathrm{mmol} / \mathrm{L}$ 硼
酸盐, $\mathrm{pH}$ 值 9.5 为最优的分离缓冲液。但在上述条 件下,由于游离 $\mathrm{BCA}$ 及复合物之间的淌度差异较 小,无法达到基线分离。

不同环糊精的疏水性空腔的内径不同，亲水性 外缘的类型也不同，可分别与不同尺寸和性质的分 析物形成包合物 ${ }^{[14]}$ 。本文采用具有包合效应的 $\beta$ $\mathrm{CD}$ 为添加剂, 利用游离 $\mathrm{BCA}$ 和 $\mathrm{BCA}-\mathrm{Cu}^{+}$复合物与 $\beta$-CD 包合作用的差异实现其有效的分离。实验结 果表明,由于包合物的形成，使得 BCA 与复合物之 间产生淌度差异 $\left(\mu_{\mathrm{BCA}-\mathrm{Cu}+-\beta-\mathrm{CD}}>\mu_{\mathrm{BCA}-\beta-\mathrm{CD}}\right)$ 。如图 4 所示, 随着 $\beta$-CD 浓度自 0 增至 $15 \mathrm{mmol} / \mathrm{L}$, 游离的 $\mathrm{BCA}$ 和复合物间的分离度 $\left(R_{\mathrm{s}}\right)$ 逐渐从 0.67 增加到 2. 94。因样品中的 BCA 淌度远大于分离缓冲液的 淌度, 由 $\mathrm{CE}$ 谱图可见游离的 $\mathrm{BCA}$ 呈现一典型的前 伸峰，从而亦略微导致 BCA- $\mathrm{Cu}^{+}$峰形前伸。

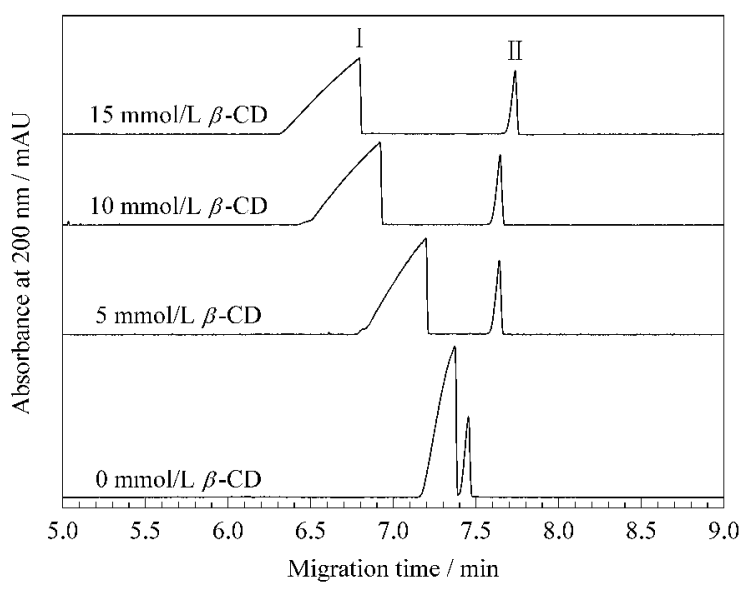

图 4 不同浓度的 $\boldsymbol{\beta}-\mathrm{CD}$ 对于游离 $\mathrm{BCA}$ 及 $\mathrm{BCA}-\mathrm{Cu}{ }^{+}$复合物分离的影响

Fig. 4 Effect of $\beta$-CD concentration on the separation of free $\mathrm{BCA}$ and $\mathrm{BCA}-\mathrm{Cu}{ }^{+}$complex

CE separation buffer : $60 \mathrm{mmol} / \mathrm{L}$ borate buffer $(\mathrm{pH} \mathrm{9.5)}$ containing $0,5,10$ or $15 \mathrm{mmol} / \mathrm{L} \beta$-CD , separately. Other conditions are the same as in Fig. $3 \mathrm{f}-1$.

Peak I : free BCA ; peak II : $\mathrm{BCA}-\mathrm{Cu}^{+}$.

\subsection{2 本文方法与常规 CE-UV 测定蛋白质时的灵} 敏度比较

通常，CE-UV 方法检测蛋白质时往往采用其末 端紫外吸收 $200 \mathrm{~nm}$ 和其特征紫外吸收 $280 \mathrm{~nm}$ 作 为检测波长。而在本方法中, 基于 $\mathrm{BCA}-\mathrm{Cu}^{+}$显色反 应，可实现可见区 $567 \mathrm{~nm}$ 处的检测，可有效地消除 基质的干扰。如图 5 所示, 对于 $100 \mathrm{mg} / \mathrm{L}$ 的转铁 蛋白, 未经 $\mathrm{BCA}-\mathrm{Cu}^{+}$显色时, 其 $200 \mathrm{~nm}$ 处的峰高值 仅有 $1.27 \mathrm{mAU}$ (见图 5a), 在 $280 \mathrm{~nm}$ 波长下更末 见到检测信号; 但应用本方法, 其 $567 \mathrm{~nm}$ 处的峰高 为 $61 \mathrm{mAU}$ (见图 5b) 增强了 47 倍。当利用 $\beta$-CD 使 $\mathrm{BCA}-\mathrm{Cu}^{+}$复合物与过量游离的 $\mathrm{BCA}$ 完全分离 
后，本方法还可直接在 $200 \mathrm{~nm}$ 处检测 $\mathrm{BCA}-\mathrm{Cu}^{+}$的 特征峰, 以间接检测蛋白质, 如 $100 \mathrm{mg} / \mathrm{L}$ 转铁蛋白 相对应的 $\mathrm{BCA}-\mathrm{Cu}^{+}$的峰高值为 $521 \mathrm{mAU}$ (见图 $5 c$ ), 是末经 BCA 处理时转铁蛋白峰高值的 410 倍。
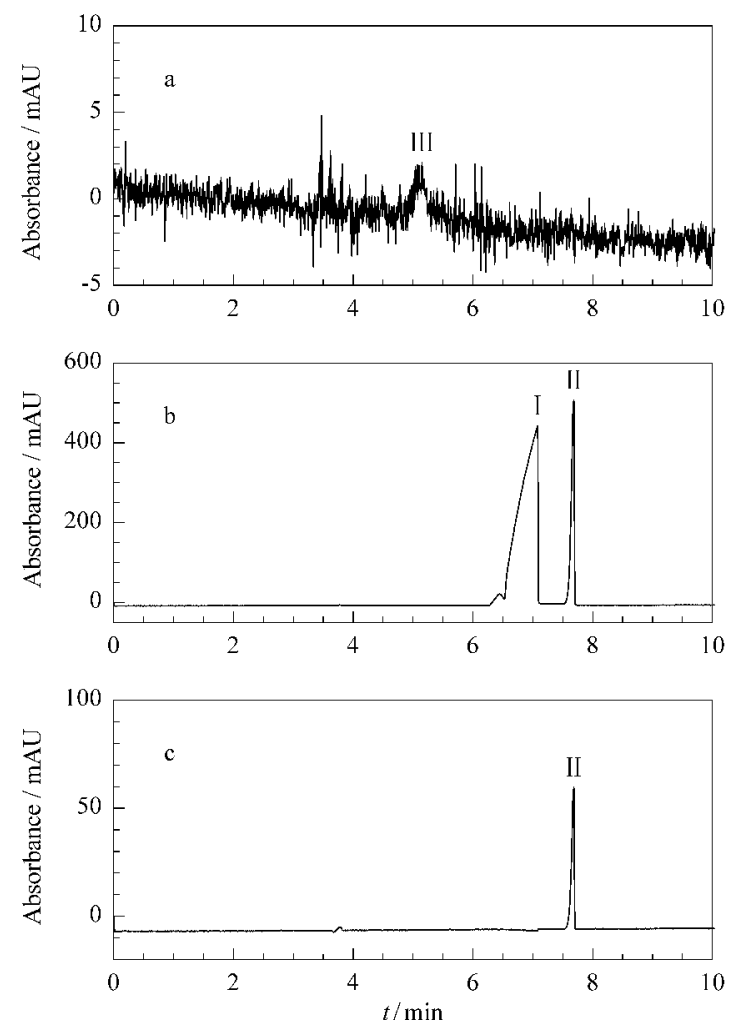

图 5 转铁蛋白经 BCA 反应前后的 $\mathrm{CE}$ 图谱

Fig. 5 Capillary electropherograms of transferrin before and after treated with $\mathrm{BCA}-\mathrm{Cu}^{+}$

a. only transferrin detected at $200 \mathrm{~nm}$; b. transferrin treated in BCA colorimetric method detected at $200 \mathrm{~nm}$; c. the same as in Fig. $5 \mathrm{~b}$ but detected at $567 \mathrm{~nm}$. The concentration of transferrin was $100 \mathrm{mg} / \mathrm{L}$. Other conditions are the same as in Fig. 3f-1.

Peak I : free BCA ; peak II : $\mathrm{BCA}-\mathrm{Cu}^{+}$; peak III : transferrin.

\section{4 线性范围及检出限}

在确立的最佳实验条件下 , $\mathrm{BCA}-\mathrm{Cu}^{+}$复合物的 峰面积与蛋白质的浓度在一定范围内呈良好的线性 关系, 转铁蛋白的线性范围为 $2 \sim 200 \mathrm{mg} / \mathrm{L}$, 线性回 归方程为 $Y=75.59+3605.33 X, r=0.9996$; 葛麻 毒素的线性范围为 $2 \sim 100 \mathrm{mg} / \mathrm{L}$,线性回归方程为 $Y=103.95+6386.62 X, r=0.9993$ 转铁蛋白和蓄 麻毒素的检出限分别为 0.33 和 $0.37 \mathrm{mg} / \mathrm{L}$ (如图 6 所示) ,而常规 BCA 法的线性范围一般介于 20 $200 \mathrm{mg} / \mathrm{L}^{[9]}$ 。

2.5 本文方法在第一届蓖麻毒素国际比对测试中 对部分样品的测定

2009 年 $10-11$ 月，我们将建立的方法应用于 德国罗伯特. 科赫研究所 (RKI) 组织的第一届蓄麻 毒素国际实验室间的比对测试工作。对 RKI 提供
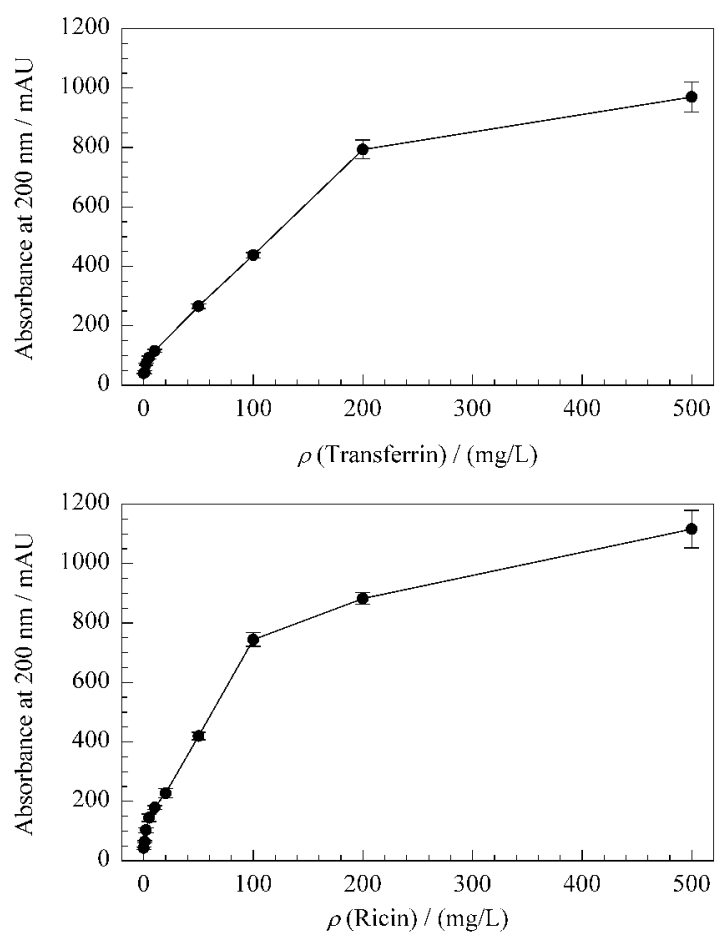

图 6 转铁蛋白和蓄麻毒素的滴定曲线

Fig. 6 Titration curves of transferrin and ricin

的 S5 样品中的蓄麻毒素含量进行了测定。将该样 品分别稀释 10,20 及 100 倍后,测得其含量为 $1022 \sim 1087 \mathrm{mg} / \mathrm{L}$, 相对标准偏差 (RSD) 为 $0.4 \%$ 6. 8\% (见表 1)。此值与 RKI 公布的真值 ( ( 1018 $\pm 243) \mathrm{mg} / \mathrm{L})$ 的符合度较高，表明了建立的方法有 较好的应用价值。

\section{表 1 本文方法在第一届蓄麻毒素国际比对测试中 对 S5 样品的测定结果 $(n=3$}

Table 1 Results of sample 5 in the First International Ricin Proficiency Test with this method $(n=3)$

\begin{tabular}{ccccc}
\hline Sample & $\begin{array}{c}\text { Dilution } \\
\text { ratio }\end{array}$ & $\begin{array}{c}\text { Measured value/ } \\
(\mathrm{mg} / \mathrm{L})\end{array}$ & $\begin{array}{c}\text { Real value/ } \\
(\mathrm{mg} / \mathrm{L})\end{array}$ & $\begin{array}{c}\text { RSD/ } \\
\%\end{array}$ \\
\hline S5-1 & 10 & $1087 \pm 165$ & & 6.8 \\
S5-2 & 20 & $1052 \pm 49$ & $1018 \pm 243$ & 3.3 \\
S5-2 & 100 & $1022 \pm 29$ & & 0.4 \\
\hline
\end{tabular}

\section{3 结论}

本文采用 $\mathrm{CE}$ 法和 $\mathrm{BCA}$ 法, 以转铁蛋白和蓄麻 毒素为模型分子, 辅以微波辅助孵育反应, 并以 $\beta$ $\mathrm{CD}$ 为有效的包合添加剂, 实现了 $\mathrm{BCA}-\mathrm{Cu}^{+}$复合物 和过量游离 $\mathrm{BCA}$ 分子的分离, 实现了在低波长 200 $\mathrm{nm}$ 下高灵敏度间接检测蛋白质的目的。该方法已 成功地应用于第一届蓄麻毒素国际比对测试，显示 了良好的应用前景。但尚需提及的是，对于多种蛋 白质混合体系，本文方法还无法达到区分的目的，此 点与 $\mathrm{BCA}$ 法自身的间接显色特性直接相关。 


\section{参考文献：}

[1] Sapan C V , Lundblad R L , Price N C. Biotechnol Appl Biochem , 1999, $29: 99$

[2] Bradford M M. Anal Biochem , $1976,72: 248$

[3] Gornall A G, Bardawill C J, David M M. J Biol Chem, $1949,177: 751$

[4] Lozzi I, Pucci A , Pantani O L, et al. Anal Biochem , 2008 , $376: 108$

[5] Walker J M. Methods Mol Biol , 1994, $32: 5$

[6] Smith P K, Krohn R I, Hermanson G T. Anal Biochem , $1985,150: 76$

[ 7 ] Yan Y, Zhang S Z , Tang J J , et al. Chinese Journal of Ana- lytical Chemistry (间妍，张淑珍，唐吉军，等。分析化学）, $2006,34(2): 187$

[8] Zhao Y F. Bioorganic Mass Spectrometry. Zhengzhou : Zhengzhou University Press (赵玉芬. 生物有机质谱. 郑州: 郑州大学出版社) , 2005:69

[9] Wiechelman K J, Braun R D, Fitzpatrick J D. Anal Biochem , $1988,175: 231$

[10] Byk G, Cohen-Ohana M, Raichman D. Biopolymers , $2006,84(3): 274$

[11] Liu J K , Lee M L. Electrophoresis , 2006, $27: 3533$

[12] Wang H , Lu M , Le X C. Anal Chem , $2005,77: 4985$

[13] Fuguet E , Reta M , Gibert C. Electrophoresis , 2008, 29 (13) : 2841

[14] Liu Y, Chen Y. Acc Chem Res ,2006, 39(10):681 